2008;10(12):1411-1420.

8. Varetti G, Guida C, Santaguida S, Chiroli E, Musacchio A. Homeostatic control of mitotic arrest. Mol Cell. 2011;44(5):710-720.

9. Holland AJ, Cleveland DW. Boveri revisited: chromosomal instability, aneuploidy and tumorigenesis. Nat Rev Mol Cell Biol. 2009;10(7):478-487.

10. Michel LS, et al. MAD2 haplo-insufficiency causes premature anaphase and chromosome instability in mammalian cells. Nature. 2001;409(6818):355-359.

11. Cimini D. Merotelic kinetochore orientation, aneuploidy, and cancer. Biochim Biophys Acta. 2008; 1786(1):32-40
12. Ganem NJ, Godinho SA, Pellman D. A mechanism linking extra centrosomes to chromosomal instability. Nature. 2009;460(7252):278-282.

13. Silkworth WT, Nardi IK, Scholl LM, Cimini D. Multipolar spindle pole coalescence is a major source of kinetochore mis-attachment and chromosome missegregation in cancer cells. PLoS One. 2009;4(8):e6564.

14. Silkworth WT, Nardi IK, Paul R, Mogilner A, Cimini D. Timing of centrosome separation is important for accurate chromosome segregation. Mol Biol Cell. 2012;23(3):401-411.

15. Araki M, et al. Centrosome protein centrin $2 /$ caltractin 1 is part of the xeroderma pigmentosum group $\mathrm{C}$ complex that initiates global genome nucleotide excision repair.J Biol Chem. 2001;276(22):18665-18672.

16. Fuchs G, et al. RNF20 and USP44 regulate stem cell differentiation by modulating $\mathrm{H} 2 \mathrm{~B}$ monoubiquitylation. Mol Cell. 2012;46(5):662-673.

17. Torres EM, et al. Effects of aneuploidy on cellular physiology and cell division in haploid yeast. Science. 2007;317(5840):916-924.

18. Williams BR, et al. Aneuploidy affects proliferation and spontaneous immortalization in mammalian cells. Science. 2008;322(5902):703-709.

19. Torres EM, et al. Identification of aneuploidy-tolerating mutations. Cell. 2010;143(1):71-83.

\title{
Mitochondrial heme: an exit strategy at last
}

\author{
Mark D. Fleming ${ }^{1}$ and lqbal Hamza ${ }^{2}$
}

1Department of Pathology, Boston Children's Hospital, Harvard Medical School, Boston, Massachusetts, USA. 2Department of Animal and Avian Sciences, Department of Cell Biology and Molecular Genetics, University of Maryland, College Park, Maryland, USA.

\begin{abstract}
The transport of heme across membranes is critical for iron absorption, the formation of hemoglobin and other hemoproteins, and iron recycling in macrophages. However, the identity of heme transport proteins has been elusive. In this issue of the JCI, Chiabrando et al. reveal that an isoform of the feline leukemia virus subgroup $\mathrm{C}$ receptor (FLVCR1) exports heme from the mitochondria and is critical for erythroid differentiation.
\end{abstract}

Nearly two-thirds of the body's iron endowment is in the form of hemoglobin in erythrocytes, and each erythrocyte contains more than a billion iron atoms in the form of heme (1). Consequently, it is not surprising that inherited or acquired defects in hemoglobin synthesis, including the thalassemias, hemoglobinopathies, and iron deficiency, are among the most prevalent human diseases. Importantly, the approximately 360 billion erythrocytes produced daily require over $250 \mathrm{mg}$ of heme to assemble into hemoglobin. Heme is synthesized in the mitochondria, but globin is translated in the cytosol, and it is unclear how newly synthesized heme is transported out of the mitochondria for incorporation into hemoglobin (2). Heme transport across membranes is important for dietary iron absorption and crucial for erythrocyte heme iron recycling in the reticuloendothelial system (RES) macrophage. Despite the physiologic importance of these processes, the molecular pathways of transmembrane heme transport have, for the most part, remained obscure, in large part due to technical difficulties in identifying heme-specific transporters in

Conflict of interest: The authors have declared that no conflict of interest exists.

Citation for this article: J Clin Invest. 2012; 122(12):4328-4330. doi:10.1172/JCI66607. mammalian cells and the inability to translate these findings to whole organisms. In this issue of the JCI, using a combination of siRNA studies and targeted mutations in mice, Chiabrando et al. provide compelling evidence that an isoform of the feline leukemia virus subgroup C receptor (FLVCR1) exports heme from the mitochondria (3).

\section{The requirement for heme transport}

For more than 50 years, it has been known that nutritional heme-iron is absorbed in the intestine by an active, energy-dependent, and inducible process that requires a heme transporter in enterocytes. This is because elemental iron has limited bioavailability in the intestine due to the presence of natural iron chelators, such as phytates and tannins, as well as its tendency to oxidize (i.e., to rust) and precipitate. In contrast, even though heme-iron constitutes only one-third of total dietary iron, it is more easily absorbed and is the source for two-thirds of body iron in meat-eating individuals (4). This is a consequence of heme's solubility at intestinal $\mathrm{pH}$ and because its uptake is not known to be influenced by other dietary factors.

Heme must also be transported across mitochondrial membranes because the final steps of heme synthesis occur in the mitochondria, but some hemoproteins such as hemoglobin are cytosolic (2). Like- wise, heme transport out of phagolysosomes is an essential component of iron recycling by macrophages, as heme oxygenase (Hmox), the enzyme that catalyzes the oxidation of heme to biliverdin, carbon monoxide, and ferric iron, is found largely tethered to the cytosolic surface of the endoplasmic reticulum (5). Iron recycling out of the macrophage is mediated by the ferrous iron exporter ferroportin (FPN1) (6), whose cell-surface expression is tightly controlled by the systemic iron regulatory hormone hepcidin (7). In this manner, iron catabolized by RES macrophages from heme can be released immediately to the plasma to replenish the $\mathrm{Fe}_{2}$-Tf pool necessary for erythropoiesis or stored as ferritin for subsequent use.

\section{Mammalian heme transporters}

The identification and characterization of heme and other porphyrin transporters in mammals has proven to be difficult (2), in part due to a lack of genetic and molecular tools, but also as a consequence of the promiscuity of proteins capable of transporting heme with low affinity. Furthermore, in vivo investigation of transporter proteins identified in vitro or on the basis of their expression pattern has been misleading or has provided ambiguous results. For example, Hcp1, a putative apical intestinal heme importer, was eventually proven to be essential for folate transport in the intestine when it was found to be mutated in patients with congenital folate deficiency $(8,9)$. Whether Hcp1 also contributes to the absorption of heme in the intestine is uncertain at this time. Some evidence suggests that the breast cancer 


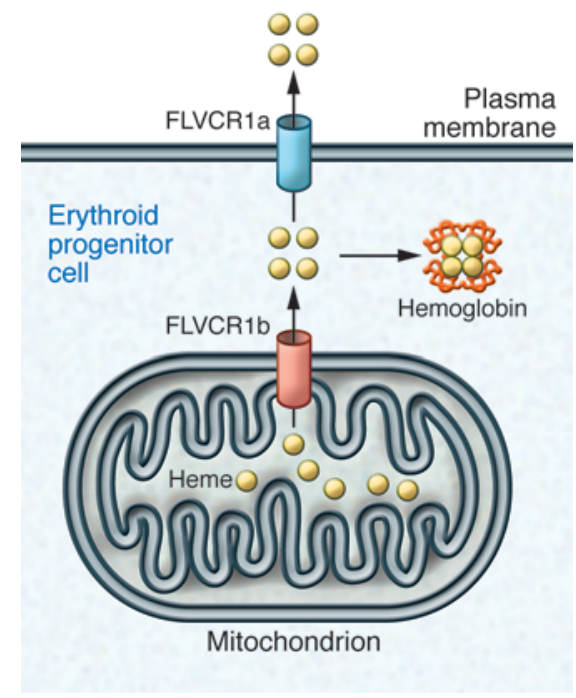

Figure 1

Two isoforms of FLVCR1 may regulate heme levels. FLVCRa isoform had previously been postulated to transport cytosolic heme across the plasma membrane. Chiabrando et al. now show that a mitochondrial isoform, FLVCR1b, transports heme from the mitochondria into the cytosol and thus is critical for the synthesis of hemoglobin and differentiation of erythroid cells.

resistance protein (BCRP, also known as ABCG2) can export heme from cells (10). However, BCRP has a broad specificity and can mediate the efflux of a large number of natural and unnatural substrates, and for that reason has been called a multidrug resistance protein (11). Furthermore, null alleles in BCRP in humans result in the "Junior" blood system group, which has no apparent clinical phenotype other than transfusion incompatibility (12). Using the heme auxotroph Caenorhabditis elegans, Hamza and colleagues identified a number of heme-responsive genes (HRGs) and their mammalian orthologs, including HRG1, which appears to transport heme with high affinity and specificity $(13,14)$. Immunolocalization studies reveal that mammalian HRG1 localizes to endolysosomes and the phagolysosome of RES macrophages $(13,15)$.

\section{Enter FLVCR1}

More than a decade ago, FLVCR1 was cloned and found to be a member of the major facilitator family of transmembrane transporters (16). Unlike other feline leukemia viruses, subgroup $C$ viruses induce not leukemia, but rather pure red cell aplasia, characterized by an absence of erythroid progenitors in the bone marrow. That the virus infected all bone marrow cells, but produced an erythroid-specific phenotype, was a clue to the function of the protein. Endogenous production of the viral capsid protein interferes with the cellular expression of FLVCR1, effectively resulting in a knockdown of the protein (17). The singular toxicity to erythroid precursors suggested that FLVCR1 performed a function uniquely essential to bone marrow cells of this lineage. Abkowitz and colleagues eventually demonstrated in vitro that FLVCR1 is a heme exporter and that targeted deletion of FLVCR1 in mice results in a severe macrocytic anemia characterized by a proerythroblast maturation arrest (18). Based upon the cell-surface localization of FLVCR1, these authors hypothesized that the phenotype is a consequence of the requirement of early erythroid precursors to export heme to avoid heme toxicity in the absence of active globin synthesis. While the murine knockout data were entirely supportive of the feline viral infection phenotype, the pathogenesis of the erythroid dysfunction remained an enigma.

\section{A mitochondrial FLVCR1 isoform}

In this issue, Emanuela Tolosano's group describes the identification of an mRNA transcript in EST databases encoding an isoform of FLVCR1 termed FLVCR1b (3). This isoform contains a shortened $\mathrm{N}$ terminus as a result of an alternative transcriptional start site in intron 2 of the previously described FLVCR1a mRNA. The protein encoded by the FLVCR $1 b$ transcript includes amino acids 277-555 of FLVCR1a and encodes a mitochondrial targeting signal at the new $\mathrm{N}$ terminus. Targeted deletion of the exon specific to FLVCR1a resulted in skeletal defects and vascular abnormalities, but not anemia, indicating that loss of the cell-surface FLVCR1a is dispensable for erythropoiesis. In contrast, siRNA-mediated knockdown of the FLVCR1b isoform impaired erythroid differentiation in vitro. While the authors do not directly demonstrate that FLVCR1b transports heme out of mitochondria, they do show that overexpression of the protein increases cytosolic heme and that knockdown of FLVCR1b results in mitochondrial heme retention (3). Taken together, these data suggest that the FLVCR1b-null phenotype may be due to cytosolic heme deficiency rather than heme accumulation, as had been originally proposed (Figure 1).

\section{FLVCR1 and human disease}

The association of pure red cell aplasia and skeletal abnormalities has suggested that mutations in FLVCR1 might be found in human patients with the inherited form of pure red cell aplasia termed DiamondBlackfan anemia (DBA), which is also associated with skeletal abnormalities. FLVCR1 mutations in DBA patients have not been found as of yet (19); however, if they were to exist, one would predict that they would occur in the FLVCR1b isoform. Interestingly, missense mutations in FLVCR1 have recently been described in patients with the rare autosomal recessive disease posterior column ataxia and retinitis pigmentosa (PCARP) $(20,21)$. Three of the four reported disease-associated FLVCR1 variants, all of which are present in the homozygous state in PCARP patients, occur in exon 1 of the gene - the part of the gene that is omitted in the FLVCR1b transcript and protein. Based on this finding, one might speculate that heme export by the cell-surface FLVCR1a may have a uniquely important function in neuronal cells. It is equally possible, as has been observed with many other proteins capable of transporting heme, that FLVCR1a binds other substrates with higher affinity than heme. It is, however, more problematic to reconcile the fourth patient mutation, which occurs in a transmembrane domain common to the FLVCR1a and FLVCR1b isoforms. Individuals harboring this mutation are not anemic, as the murine model would predict (20), raising the possibility that this genetic lesion has a unique effect on transport of an alternative substrate. If FLVCR1b localizes to the inner membrane of the mitochondria, does heme exit the mitochondrial outer membrane through unconventional means? Furthermore, how do eukaryotes that lack obvious FLVCR homologs, such as yeast, export heme from the mitochondria? Pertinent biochemical questions also remain: FLVCR1a is proposed to transport heme out of the cytoplasm, while FLVCR1b transports heme into the cytoplasm. Because FLVCR is an MFS family member of secondary transporters, are the sources of energy to translocate heme and the kinetic mechanisms (uniporter, symporter or antiporter) different between the two isoforms? Only time will reveal the truth in the myriad of theoretical possibilities.

Address correspondence to: Mark D. Fleming, Department of Pathology, Boston Children's Hospital, S. Burt Wolbach 
Professor of Pathology, Harvard Medical School, Bader 124.1, 300 Longwood Ave., Boston, Massachusetts 02115, USA. Phone: 617.919.2664; Fax: 617.730.0168; E-mail: mark.fleming@childrens.harvard.edu.

1. Knutson M, Wessling-Resnick M. Iron metabolism in the reticuloendothelial system. Crit Rev Biochem Mol Biol. 2003;38(1):61-88.

2. Severance S, Hamza I. Trafficking of heme and porphyrins in metazoa. Chem Rev. 2009; 109(10):4596-4616.

3. Chiabrando D, et al. The mitochondrial heme exporter FLVCR1b mediates erythroid differentiation. J Clin Invest. 2012;122(12):4569-4579.

4. Uzel C, Conrad ME. Absorption of heme iron. Semin Hematol. 1998;35(1):27-34.

5. Yanatori I, Tabuchi M, Kawai Y, Yasui Y, Akagi R, Kishi F. Heme and non-heme iron transporters in non-polarized and polarized cells. BMC Cell Biol. 2010;11:39.

6. Donovan A, et al. Positional cloning of zebrafish ferroportin 1 identifies a conserved vertebrate iron exporter. Nature. 2000;403(6771):776-781.

7. Nemeth E, et al. Hepcidin regulates cellular iron efflux by binding to ferroportin and inducing its internalization. Science. 2004;306(5704):2090-2093.

8. Qiu A, et al. Identification of an intestinal folate transporter and the molecular basis for hereditary folate malabsorption. Cell. 2006;127(5):917-928.

9. Salojin KV, et al. A mouse model of hereditary folate malabsorption: deletion of the PCFT gene leads to systemic folate deficiency. Blood. 2011; 117(18):4895-4904

10. Krishnamurthy $P$, et al. The stem cell marker Bcrp/ABCG2 enhances hypoxic cell survival through interactions with heme. J Biol Chem. 2004; 279(23):24218-24225.

11. Robey RW, Ierano C, Zhan Z, Bates SE. The challenge of exploiting ABCG2 in the clinic. Curr Pharm Biotechnol. 2011;12(4):595-608.

12. Saison C, et al. Null alleles of ABCG2 encoding the breast cancer resistance protein define the new blood group system Junior. Nat Genet. 2012; 44(2):174-177.

13. Rajagopal A, et al. Haem homeostasis is regulated by the conserved and concerted functions of HRG-1 proteins. Nature. 2008;453(7198):1127-1131.

14. Yuan X, Protchenko O, Philpott CC, Hamza I. Topologically conserved residues direct heme transport in HRG-1-related proteins. J Biol Chem.
2012;287(7):4914-4924.

15. Delaby C, et al. Subcellular localization of iron and heme metabolism related proteins at early stages of erythrophagocytosis. PloS One. 2012;7(7):e42199.

16. Quigley JG, et al. Cloning of the cellular receptor for feline leukemia virus subgroup C (FeLV-C), a retrovirus that induces red cell aplasia. Blood. 2000;95(3):1093-1099.

17. Khan AA, Quigley JG. Control of intracellular heme levels: heme transporters and heme oxygenases. Biochim Biophys Acta. 2011;1813(5):668-682.

18. Keel SB, et al. A heme export protein is required for red blood cell differentiation and iron homeostasis. Science. 2008;319(5864):825-828.

19. Quigley JG, Gazda H, Yang Z, Ball S, Sieff CA, Abkowitz JL. Investigation of a putative role for FLVCR, a cytoplasmic heme exporter, in Diamond-Blackfan anemia. Blood Cells Mol Dis. 2005; 35(2):189-192.

20. Ishiura $\mathrm{H}$, et al. Posterior column ataxia with retinitis pigmentosa in a Japanese family with a novel mutation in FLVCR1. Neurogenetics. 2011; 12(2):117-121.

21. Rajadhyaksha AM, et al. Mutations in FLVCR1 cause posterior column ataxia and retinitis pigmentosa. Am J Hum Genet. 2010;87(5):643-654.

\title{
Taking a bite: endocytosis in the maintenance of the slit diaphragm
}

\author{
Rosemary V. Sampogna and Qais Al-Awqati
}

Department of Medicine, College of Physicians and Surgeons of Columbia University, New York, New York, USA.

\begin{abstract}
In the kidney, the slit diaphragm joins adjacent podocytes, forming an epithelial barrier that filters plasma into the urinary space, yet retains blood cells and proteins within the circulation. In this issue of the JCI, Soda et al. have identified clathrin-mediated endocytosis as a central mechanism by which the function and structural integrity of the slit diaphragm are maintained.
\end{abstract}

The glomeruli of the human kidney filter almost 200 liters of fluid every day, but must retain the albumin and other large proteins within the circulation. The fluid has to cross three layers: the endothelium, the glomerular basement membrane (GBM), and the epithelial cells or podocytes. The GBM (1) and the slit diaphragm (2), a structure that joins adjacent podocytes, are two barriers to protein filtration, but the endothelial cell also likely plays a role. Disruption of these barriers results in proteinuria, a consequence of many kidney diseases.

Podocytes begin life as tall epithelial cells that have typical tight junctions near the apex separating the apical surface from the lateral cell membranes. As development

Conflict of interest: The authors have declared that no conflict of interest exists.

Citation for this article: J Clin Invest. 2012; 122(12):4330-4333. doi:10.1172/JCI65785. progresses, these junctions migrate downward toward the basal surface of the mature podocytes, becoming the slit diaphragm (3). Unlike other polarized epithelia, the lateral surface is above these specialized tight junctions and is continuous with and identical to the apical surface. The basal surface differentiates into thin interdigitating structures called foot processes that are joined together by the cell-cell junctions of the slit diaphragm. During proteinuric disease, the foot processes lose their fine structure and collapse into a mass of cytoplasm on top of the basement membrane (4). The observation that a great abundance of actin and actin-binding proteins accumulates in these diseased foot processes suggested that actin dysregulation might play a key role in the pathogenic progression (5). The discovery that $\alpha$-actinin 4 mutations were also associated with heavy proteinuria also helped bring attention to the role of actin (6).
Since then, mutations in humans or animals in other actin-binding proteins including Nck, CD2AP, and dynamin were also found to mediate proteinuria (7). Further, the cytoplasmic domains of many slit diaphragm components can bind actin or signal to actin, further suggesting a key role for this cytoskeletal protein (8). But what was the actual cell biological process in which actin was so central? Was the primary function of actin to maintain the slit diaphragm, or was the actin rearrangement merely a consequence of the permeability change? Here, one might turn to basic cell biology and physiology to find the definitive answers. Unfortunately, the podocyte, when grown in culture, loses its complex shape; it has neither foot processes nor a real slit diaphragm. Given that actin is directly involved in cell shape determination and maintenance, this has placed the field in a difficult position. However, an article in this issue by Soda et al. has provided the first insight into the process of maintenance of the slit diaphragm (9). The authors show that clathrinmediated endocytosis is required for podocyte function and is the likely effector of the changes in actin that are so often observed. 